\title{
Pérez de la Fuente, Oscar; Falcão, Monique y Oliva Martínez, J. Daniel (eds.) Struggles for Recognition. Cultural Pluralism and Rights of Minorities. Madrid: Dykinson, 2020.
}

Jesús Mora

Universidad Carlos III de Madrid

Fecha de recepción 27/05/2021 I De publicación: 23/12/2021

Desde hace alrededor de tres décadas, los debates político-filosóficos sobre la justicia han pivotado en torno a dos ideas fundamentales: la redistribución y el reconocimiento. Como consecuencia de esos debates, aparecen dos corrientes con importantes - y en ocasiones insalvables — discrepancias sobre la forma y el contenido verdadero de la justicia: las teorías de la redistribución y las teorías del reconocimiento. Estas últimas emergen para reivindicar la diferencia como valor esencial para la justicia $\mathrm{y}$, en parte, como una crítica a algunas implicaciones de las concepciones liberales que surgen de la Ilustración y que, desde su punto de vista, habían dado lugar a un ideal unívoco de ser humano, un ideal que se consideraba incapaz de tener en cuenta la diversidad de expresiones culturales presente en la práctica totalidad de los estados modernos. Esa reivindicación de la diversidad está estrechamente conectada con los derechos de las minorías culturales y sus luchas por el reconocimiento social e institucional a su especificidad como grupos diferenciados de la sociedad mayoritaria.

En Struggles for Recognition. Cultural Pluralism and Rights of Minorities, Oscar Pérez de la Fuente, Monique Falcão y J. Daniel Oliva Martínez recogen las contribuciones de diversos autores acerca de temas esenciales para los debates sobre el reconocimiento, los derechos de las minorías y el pluralismo cultural. Dichas contribuciones fueron presentadas originalmente en un seminario en línea con título idéntico al del libro, que se celebró a mediados de 2020 bajo el amparo del Instituto de Derechos Humanos Gregorio Peces-Barba y el Departamento de Derecho Internacional, Ecelsiástico y Filosofía del Derecho de la Universidad Carlos III de Madrid, la Universidade Santa Ursula de Río de Janeiro y el Comité de Investigación 26 sobre Derechos Humanos de la Asociación Internacional de Ciencia Política (IPSA). El libro está dividido en cinco secciones, que sirven para estructurar los capítulos que componen la obra en varios ejes temáticos: minorías e inmigración; nacionalismo, reconocimiento y libertad de 
expresión; derechos de los animales y aplicación intercultural del derecho; derecho indígena y derechos indígenas; $\mathrm{y}$, por último, pueblos indígenas y derechos naturales.

La primera sección, sobre minorías e inmigración, comienza con el capítulo “The Persistent Oppression of Roma in the Legal Field: from Outlaws to Epistemic Violence”, en el que Marc H. Vallés e Ida Nafstad exploran distintas instancias de violencia epistémica - situaciones en las que una parte de la audiencia no reconoce a la otra como un interlocutor válido - sufridas por la población romaní en los países escandinavos. A esta contribución le sigue "The Tightrope of Legitimacy and Illegality in the Political Mobilisation of Migrants in Sâo Paulo", de Augusto Veloso Leão, que aborda el ejercicio del derecho a la movilización política por parte de las personas migrantes en Brasil y, en concreto, en la ciudad de São Paulo. El autor reflexiona, además, sobre las consecuencias de las movilizaciones que derivaron de los casos de Zulmira de Sousa y Brayan Capcha, ambos migrantes asesinados. En la tercera contribución de la sección, "Children and Digital Rights Effectiveness. Some Reflections about the Right to Access to the Internet", Stefania Attolini analiza algunas cuestiones fundamentales, en términos de derechos humanos, relativas al acceso a Internet en la infancia, como el analfabetismo y la brecha digital, la educación en línea o la privacidad y la seguridad de los contenidos.

La Sección II, sobre nacionalismo, reconocimiento y libertad de expresión, arranca con “A Comparative Analysis of the Different Paradigms of Nationalism and the Nation", de Guillermo Reyes Pascual. En este capítulo, el autor analiza los paradigmas clásicos y contemporáneos del nacionalismo y establece un diálogo entre ellos en el que compara algunos elementos comunes, pero también algunas discrepancias esenciales. La cuestión del nacionalismo se conecta con el reconocimiento a las minorías en la siguiente contribución, "Mechanisms of National Minority Recognition in Post-Soviet States. Cases of Kazakhstan, Lithuania, and Russia”. Con ella, Aziz Berdiqulov presenta una aproximación al estatus legal de las minorías étnicas en tres países - Kazajstán, Lituania y Rusia — en los que no existe un procedimiento legal que permita, precisamente, otorgar a un determinado grupo el estatus jurídico de minoría y los derechos y mecanismos de protección que ello conlleva. El tema central del siguiente capítulo, "The Cimbrians and their "Law": A Possibility of Longobard Laws Continuum", a cargo de Gustavo Baretieri, es el pluralismo legal. El autor evalúa la presencia simultánea de varias tradiciones jurídicas en un mismo estado, con todo lo que ello conlleva, a partir del caso del derecho lombardo en el norte de Italia, y ofrece algunas nociones interesantes sobre la evolución del concepto del derecho, desde perspectivas más vinculadas a las costumbres comunitarias y el derecho natural, hacia otras más 
vinculadas con la acción totalizadora del Estado como autoridad suprema. El siguiente capítulo, "On Recognition", a cargo de Oscar Pérez de la Fuente, sirve como repaso de los pilares teóricos en los que se apoyan muchos de los debates sobre el reconocimiento presentes en la obra, pero con la mirada puesta en el problema concreto del discurso del odio. El autor analiza las aproximaciones a la cuestión del reconocimiento de tres de los teóricos más destacados en este ámbito en los últimos años: Axel Honneth, Charles Taylor y Nancy Fraser. Y, junto a ese análisis, propone algunas reflexiones sobre el encaje de la noción de discurso de odio en sus concepciones y, en particular, en las ideas de cada uno de ellos sobre las razones que explican la importancia del reconocimiento. En la contribución que cierra la sección, Tatiana Barandova analiza la persecución de actos performativos críticos con la religión en Rusia como actos de “blasfemia”. Con el título “'On-Going Blasphemy' or Pussy Riot Casus Echo via Argumentation Worlds", el capítulo presenta una detallada explicación del contexto de la persecución de actos blasfemos en Rusia, desde las recientes reformas constitucionales hasta la tradición de reivindicaciones frente a la Iglesia Ortodoxa promovida desde hace décadas por distintos grupos, algunos de ellos compuestos mayoritariamente por mujeres. Y, con ese trasfondo, reflexiona sobre la persecución general al grupo Pussy Riot y las ramificaciones de ese caso en la deriva de Rusia hacia un régimen jurídico menos permisivo con las críticas a la Iglesia Ortodoxa.

La Sección III de la obra aborda la aplicación intercultural del derecho, así como sus implicaciones para los derechos de los animales. Con "Cultural Diversity under the Scope of Criminal Law: the Colombian Case", Olga Lucía Abello Restrepo inaugura la sección analizando la tensión entre derechos fundamentales como la vida, la libertad y la igualdad, por un lado, y el respeto a los derechos de las minorías, por el otro. En concreto, la autora evalúa las contradicciones entre la plena satisfacción de esos derechos y la idea de crímenes culturalmente motivados: conductas por las que la sociedad mayoritaria penaliza a una minoría por un comportamiento que, en esa minoría, no merece reproche legal alguno. El segundo capítulo de la sección se titula "About the Need to Incorporate an Intercultural Perspective in the Application of Pretrial Detention: Notes from the Baguazo Case". En él, Vanessa CuentasPortocarrero defiende la incorporación de una perspectiva intercultural al derecho procesal penal de Perú $\mathrm{y}$, en concreto, a los casos de detenciones previas al juicio, que en los últimos tiempos han dado lugar a casos con consecuencias especialmente lesivas para los miembros de algunas comunidades indígenas. Los debates sobre los derechos de los animales aparecen por primera vez en el libro con "Analysis of Nonhuman Animals' Status in Urban Areas Through the Biocentric Paradigm: Institutionalizing a Representative Mechanism to Provide Recognition of the Animal as a Subject of the City", de Giselle 
Maria Custódio Cardoso y Emerson Affonso da Costa Moura. En este capítulo, los autores presentan las perspectivas contractualistas de John Rawls y Martha Nussbaum y estudian sus implicaciones para los derechos de los animales. Además, exponen el paradigma del biocentrismo y defienden la necesidad de mecanismos de protección o tutela para que diferentes personas y colectivos puedan actuar como representantes de los animales en todos aquellos procedimientos jurídicos relevantes para salvaguardar sus intereses. En una línea similar, Monique Falcâo, Nattiele Gomes, Thiago Prado y Julia Roehrs Canto presentan el último capítulo de la sección, titulado "Animals as Minorities. Contributions from Civil Society to the Effectiveness of Rights". En él, los autores se refieren a la intervención de algunos colectivos animalistas en sentencias favorables a los derechos de los animales y a cómo esas decisiones pueden afectar al discurso mayoritario sobre los animales, así como a la interpretación judicial de la Constitución de Brasil en relación con este asunto.

La cuarta sección se centra en los sistemas legales indígenas y los derechos de las personas y comunidades indígenas. En "Peoples and Persons in the UNDRIP", Dwight Newman analiza la Declaración de las Naciones Unidas sobre los Derechos de los Pueblos Indígenas, y defiende la necesidad de un enfoque híbrido que aborde los derechos indígenas tanto en términos individuales como colectivos. En esta línea, el autor critica las interpretaciones de la declaración que han realizado algunos estados y que, por diversas razones, suelen omitir la importancia que concede el texto a los derechos colectivos. Frente a esas interpretaciones, el autor propone un análisis de la declaración como un documento que combina una preocupación, tanto por los derechos individuales de las personas indígenas como por sus derechos colectivos como miembros de comunidades. En la siguiente contribución, titulada "A Critical Analysis of the Proposed Amendment to the Brazilian Constitution No 215/2000: Demarcation of Indigenous Lands", Pedro Curvello Saavedra Avzaradel y Camila Graça Camatta repasan el estatus jurídico actual de los derechos sobre la tierra de algunas comunidades indígenas y afrodescendientes de Brasil. En concreto, abordan la propuesta de reforma constitucional 215/2000 y sus implicaciones para el procedimiento de demarcación, que sirve para dar cobertura legal a los derechos de algunas comunidades con respecto a ciertas tierras sobre las que no ostentan títulos formales de propiedad. En el capítulo, los autores concluyen que la reforma analizada debería considerarse inconstitucional por sus implicaciones negativas para la separación de poderes y los derechos de estas comunidades reconocidos en el texto constitucional brasileño. María Cruz la Chica firma el siguiente capítulo, titulado "The Responsibility of the States in the Current Situation of Women's Rights in Indigenous Normative Systems in Latin America”. La autora denuncia el silencio en los estudios indígenas sobre las diferentes 
formas de violencia y opresión a las que se enfrentan las mujeres en esas comunidades. En ese sentido, repasa algunas instancias de violencia habituales y analiza los diferentes obstáculos que afrontan las mujeres indígenas que desean denunciarlas. Con el siguiente capítulo de la sección, "Ethnic Minorities in Brazil. Some Conceptual Aspects”, Monique Falcão pretende aclarar algunas diferencias y similitudes entre dos comunidades muy relevantes en Brasil: los quilombolas y los pueblos indígenas brasileños. En el caso de los primeros, Falcão resalta su conexión identitaria con la tierra, vinculada a la ruptura con su condición de esclavos en el pasado. En el caso de los segundos, destaca los mecanismos legales para el reconocimiento de estas comunidades y la garantía de sus derechos, también muy vinculados a la tierra y a los recursos naturales. El último capítulo de la sección es "Food Security of Indigenous Peoples: Introduction to the Right to Cultural Food as a Legal Obligation", de Chiaretta Giordano, en el que la autora desarrolla el derecho a la alimentación cultural como necesidad esencial de las comunidades indígenas para garantizar su seguridad alimentaria. Además, la autora explora la comida como un elemento clave en la construcción de la identidad individual y colectiva para subrayar la importancia del derecho a la alimentación cultural en un contexto de migraciones cada vez más crecientes hacia el exterior de las comunidades indígenas.

La quinta y última sección del libro, sobre pueblos indígenas y recursos naturales, está compuesta por dos capítulos. El primero, a cargo de Valentina de Gregorio, Giulia Parola, Arianna Porrone, Margherita Paola Poto y Apostolos Tsiouvalas, analiza la capacidad del derecho indígena para promover aprendizajes reseñables para el derecho occidental. Bajo el título "Learning from the Indigenous Law: the Core Values of Inclusion, Coexistence and Resilience", los autores conectan ese aprendizaje con tres valores fundamentales para las comunidades indígenas: la inclusión, la coexistencia y la resiliencia. Cada uno de esos valores se conecta, respectivamente, con la experiencia de las comunidades de los ribeirinhos, en Brasil, los Sámi, en Noruega, y las comunidades del Ártico que, en los últimos años, han visto cómo aumentaba el sufrimiento de su entorno natural y de los recursos presentes en él. Precisamente una de esas comunidades, los Sámi, es la protagonista del segundo capítulo de la sección, que además clausura esta obra: "Submarine Tailings Disposal in Arctic Indigenous Waters: A Human Rights Perspective on Nussir Mine's Impacts on the Sámi Use of Repparfjord, Norway”, de Apostolos Tsiouvalas. En ella, el autor explica las consecuencias que tienen los vertidos tóxicos realizados por compañías mineras en aguas tradicionalmente destinadas a la pesca de la comunidad Sámi. Además, Tsiouvalas lamenta algunas omisiones en la legislación noruega a la hora de proteger los intereses de esta comunidad y, en particular, el hecho de que sea el Estado quien tiene habitualmente la última palabra 
cuando se trata de conceder permisos a las compañías mineras para realizar vertidos en aguas tradicionalmente trabajadas por los Sámi.

Esta sección sobre las relaciones de los pueblos indígenas con los recursos naturales pone el punto final a una obra cuyas contribuciones trazan un recorrido muy completo a lo largo y ancho de las demandas de reconocimiento y de las principales problemáticas a las que se enfrentan las minorías culturales. La inclusión de temas como la propiedad de la tierra, las consecuencias de los delitos de odio sobre los individuos pertenecientes a minorías, las garantías del proceso penal, la alimentación o las relaciones de estas comunidades con la naturaleza presentan, además, algunas cuestiones fundamentales para las minorías que no tienen que ver solo con su estatus, sino también con sus necesidades materiales. En este sentido, la obra puede ser una herramienta útil para trazar puentes entre los dos paradigmas a los que se hizo referencia al principio de esta reseña: la redistribución y el reconocimiento. Y es que, en muchas ocasiones, lejos de ser compartimentos estancos, redistribución y reconocimiento son esferas interconectadas, hasta el punto de que sufrir discriminación con respecto a una de ellas puede dar lugar, también, a instancias de discriminación con respecto a la otra. Por eso, pese al lugar que se concede a las luchas por el reconocimiento en el título mismo de la obra, es importante destacar que tiene la virtud de poder resultar interesante, también, a lectores preocupados por los problemas materiales que afrontan las minorías en nuestras sociedades. Asimismo, la obra combina análisis teóricos rigurosos sobre algunas de las perspectivas del reconocimiento más relevantes con estudios de casos prácticos que sirven para explicar la relevancia de las luchas por el reconocimiento a partir de conflictos políticos de actualidad. Por ello, en un contexto en el que los debates sobre el reconocimiento estarán cada vez más presentes en la agenda política de nuestras sociedades, Struggles for Recognition. Cultural Pluralism and Rights of Minorities puede ser una guía muy útil para entender las reivindicaciones de las minorías culturales en el futuro, así como para analizar de forma crítica el peso de esas reivindicaciones frente a consideraciones alternativas, como la necesidad de preservar otros derechos que puedan entrar en conflicto con ellas. 